\title{
Quantitative podocyte parameters predict human native kidney and allograft half-lives
}

\author{
Abhijit S. Naik, ${ }^{1}$ Farsad Afshinnia, ${ }^{1}$ Diane Cibrik, ${ }^{1}$ Jeffrey B. Hodgin, ${ }^{2}$ Fan Wu, ${ }^{3}$ Min Zhang, ${ }^{3}$ \\ Masao Kikuchi, ${ }^{1}$ Larysa Wickman, ${ }^{4}$ Milagros Samaniego, ${ }^{1}$ Markus Bitzer, ${ }^{1}$ Jocelyn E. Wiggins, ${ }^{1}$ \\ Akinlolu Ojo, ${ }^{1}$ Yi Li, ${ }^{3}$ and Roger C. Wiggins ${ }^{1}$ \\ 'Department of Internal Medicine, ${ }^{2}$ Department of Pathology, ${ }^{3}$ School of Public Health, and ${ }^{4}$ Department of Pediatrics \\ and Communicable Diseases, University of Michigan, Ann Arbor, Michigan, USA.
}

\begin{abstract}
BACKGROUND. Kidney function decreases with age. A potential mechanistic explanation for kidney and allograft half-life has evolved through the realization that linear reduction in glomerular podocyte density could drive progressive glomerulosclerosis to impact both native kidney and allograft half-lives.
\end{abstract}

METHODS. Predictions from podometrics (quantitation of podocyte parameters) were tested using independent pathologic, functional, and outcome data for native kidneys and allografts derived from published reports and large registries.

RESULTS. With age, native kidneys exponentially develop glomerulosclerosis, reduced renal function, and end-stage kidney disease, projecting a finite average kidney life span. The slope of allograft failure rate versus age parallels that of reduction in podocyte density versus age. Quantitative modeling projects allograft half-life at any donor age, and rate of podocyte detachment parallels the observed allograft loss rate.

CONCLUSION. Native kidneys are designed to have a limited average life span of about 100-140 years. Allografts undergo an accelerated aging-like process that accounts for their unexpectedly short half-life (about 15 years), the observation that older donor age is associated with shorter allograft half-life, and the fact that long-term allograft survival has not substantially improved. Podometrics provides potential readouts for these processes, thereby offering new approaches for monitoring and intervention.

FUNDING: National Institutes of Health.

Authorship note: A.S. Naik and F. Afshinnia contributed equally to this work.

Conflict of interest: The authors have declared that no conflict of interest exists.

Submitted: February 9, 2016

Accepted: April 19, 2016

Published: May 19, 2016

Reference information:

JCI Insight. 2016;1(7):e86943.

doi:10.1172/jci.insight.86943.

\section{Introduction}

Podocytes must completely cover the filtration surface area with foot processes to maintain the glomerular filtration barrier. Failure to achieve this task due to reduced podocyte number, size, or function, or increased glomerular volume (quantitated by "podometric" methodology), results in progressive glomerular dysfunction, causing proteinuria and glomerulosclerosis and ultimately leading to end-stage kidney disease (ESKD) (1-4). Well-accepted evidence from model systems, human genetic causes for the focal segmental glomerulosclerosis (FSGS) phenotype, and observational studies in human and experimental glomerular diseases all strongly indicate that podocyte depletion per se causes glomerulosclerosis, that glomerulosclerosis causes nephron loss, and that cumulative nephron loss ultimately causes ESKD (1-16). Collectively, these data support the concept that normal podocyte density and function are necessary to maintain normal glomerular structure and function.

We recently reported that glomerular aging is associated with a progressive linear reduction in podocyte density, eventually resulting in critical podocyte depletion, triggering mass podocyte detachment, glomerular tuft collapse, and global glomerulosclerosis (17). Podocyte density decreasing linearly with age would be predicted to cause accelerating global glomerulosclerosis, with a parallel accelerating decrease in renal function and increasing ESKD incidence. This scenario also predicts that human kidneys will have a finite measurable half-life. All glomerular diseases have increased rates of podocyte detachment and can 
Podocyte data sources

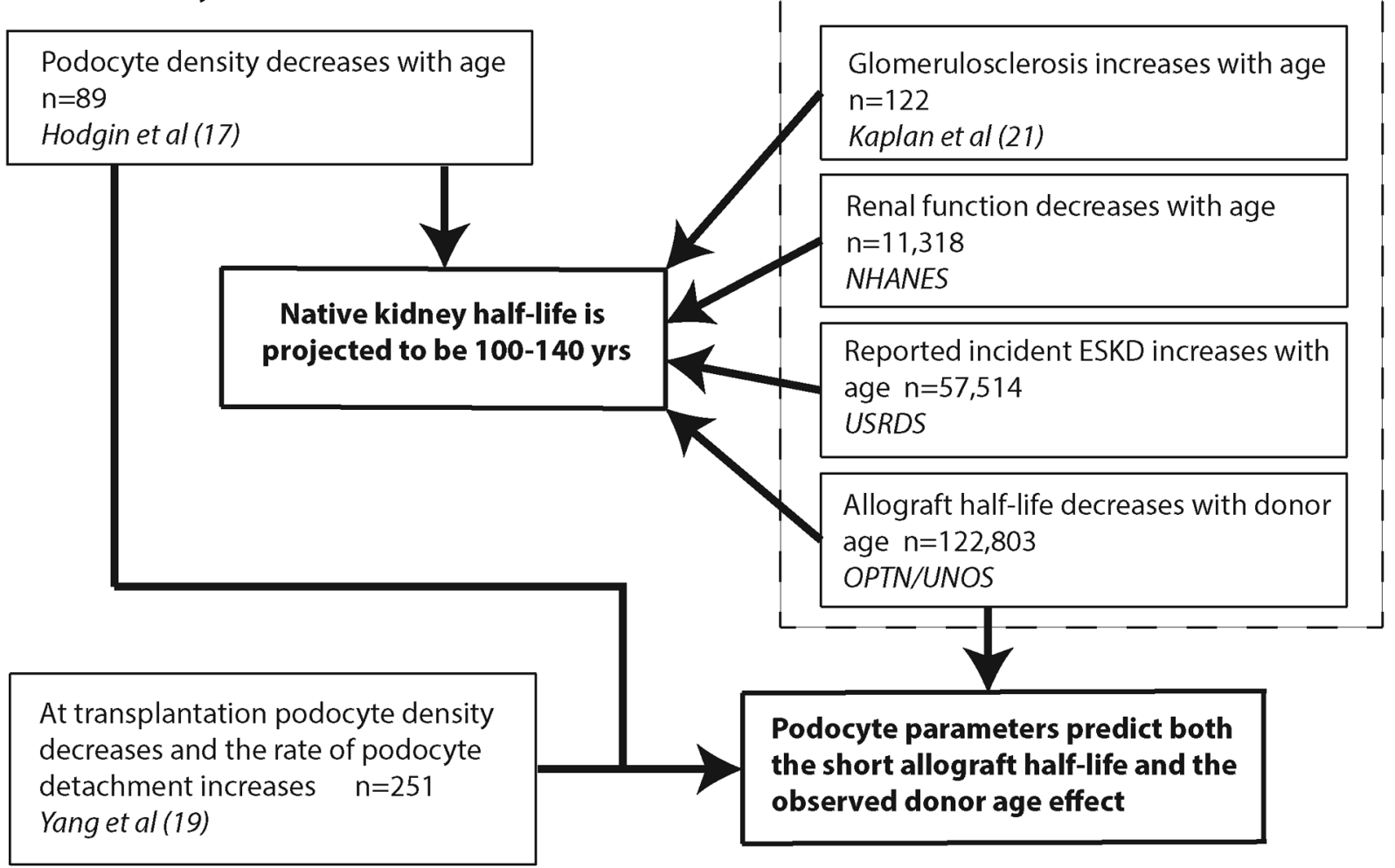

Figure 1. Overview of data sources. Podocyte data were derived from two recent reports dealing with podocytes in normal human aging (17) and in kidney transplantation (19). Predictions derived from podocyte data were tested using other data sources, including glomerulosclerotic changes with age (21), changing renal function with age (National Health and Nutrition Examination Survey [NHANES] (22, 23), incident end-stage kidney disease (ESKD; United States Renal Data System [USRDS]; ref. 24), and allograft outcome (Organ Procurement and Transplantation Network [OPTN]/United Network for Organ Sharing (UNOS) Standard Transplant and Research [STAR]; ref. 25) data sets.

therefore be considered as superimposed accelerators of this normal underlying aging process (18). In a separate report, we found that podocyte density reduction and increased podocyte detachment rate also occur in kidney allografts, compatible with the hypothesis that reduced podocyte density could also signal allograft failure (19). We therefore explored these predictions to determine whether they are supported by available outcomes data, and with the purpose of determining why allograft half-life is so unexpectedly short compared with native kidney half-life, and so powerfully related to donor age (20).

\section{Results}

Figure 1 provides an overview of the sources of data used in this report. Figure 2 shows the previously reported podocyte density decreasing linearly with age that underpins the concepts explored in this report (17). The logical extension of the observation is that as aging proceeds, an exponentially increasing proportion of glomeruli will become globally scarred in association with accelerating reduction in renal function and prevalence of ESKD. It also suggests that kidneys will have a finite half-life, which, from podocyte density measurements, will be in the range 85-130 years. These hypotheses were tested using data from published studies and large registries as shown in Figure 1.

Native kidney aging. The gold standard study by Kaplan and colleagues (21) reported the prevalence of globally sclerotic glomeruli in autopsied kidneys using a large glomerular sample per patient (rather than a needle biopsy sample) and representative of the general population over a wide age range. Although there was wide individual variation in the presented data, this report appears to show an exponential increase in glomerulosclerosis with age (21). When these data are averaged and re-plotted as shown in Figure $3 \mathrm{~A}$, the mean global glomerulosclerosis rate does accelerate in relation to age, such that on a log plot the data fall on a straight line (Figure 3B). The projected age at which $95 \%$ of glomeruli would, on average, be sclerotic (and thereby the kidney would be at or close to end stage) is about 116 years. Since sclerotic glomeruli in 


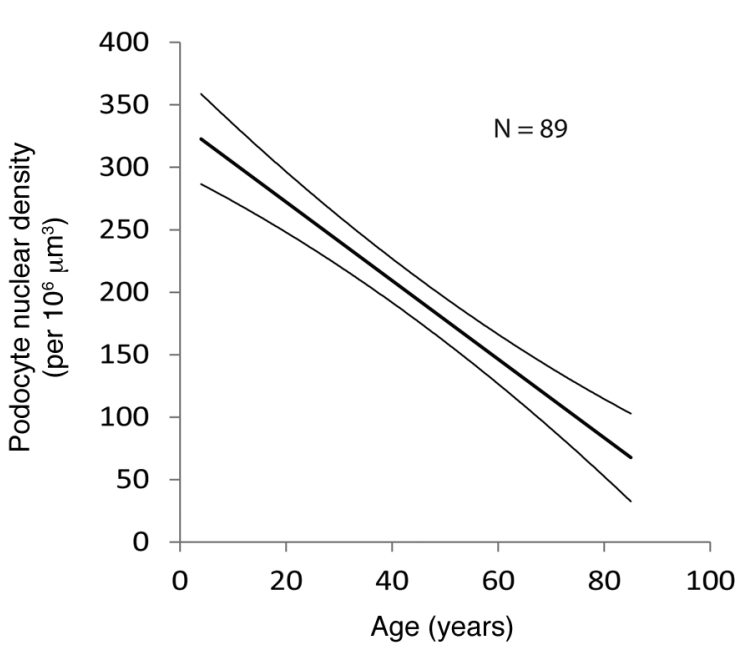

Figure 2. Linear decrease in podocyte density with age. The data shown are the median and $95 \%$ confidence limits for the reduction in podocyte density with age in human glomeruli as previously reported (17). The characteristics of the slope are given by the equation $y=-3.17$ $x$ age +338 , projecting that the age at which $y=0$ will be 107 years.

a kidney section are smaller and more difficult to identify than normal glomeruli, and may involute over time to become unrecognizable, this approach will probably underestimate the true proportion of globally scarred glomeruli and therefore may overestimate projected kidney half-life.

The National Health and Nutrition Examination Survey (NHANES) dataset $(22,23)$ was used to derive estimated glomerular filtration rate (eGFR) by a method that does not correct for age (creatinine clearance per $1.73 \mathrm{~m}^{2}$ of body surface area), as shown in Figure 3, C and D. eGFR was found to decrease in an accelerating fashion with age independent of the presence or absence of diabetes and/or hypertension. We assumed that an eGFR value of $5 \mathrm{ml} / \mathrm{min}$ per $1.73 \mathrm{~m}^{2}$ is equivalent to ESKD. A quadratic polynomial equation best fitted the curves (see Supplemental data; supplemental material available online with this article; doi:10.1172/jci.insight.85608DS1) for both "all subjects" and "only those without diabetes or hypertension." The mean age at which $5 \mathrm{ml} / \mathrm{min}$ per $1.73 \mathrm{~m}^{2}$ (ESKD) was reached was projected to be 107 (range, 103-113) years for "all subjects" and 110 (range, 102-123) years for "all subjects excluding those with diabetes or hypertension." In the case of renal function, the true relationship between loss of function and time tends to be masked by compensatory hypertrophy of remaining nephrons until such time as the capacity for further hypertrophy is exhausted. Nevertheless, the decrease in renal function with age clearly starts early and accelerates, projecting a finite kidney half-life.

Analysis of United States Renal Data System Report (USRDS) data showed that incident reported treated ESKD rate per million of population increases exponentially with age (Figure 3E; ref. 24). The cumulative reported incident treated ESKD plotted on a log scale projects a value for native kidney half-life (projected age at which 50\% of the population would have developed ESKD) at about 140 years (Figure $3 \mathrm{~F}$ ). This value is an overestimate of true native kidney half-life, because a proportion of the ESKD population will not be treated for ESKD and therefore will not be counted by the USRDS (including those dying from cardiovascular disease and/or in an ICU setting, and those who elect not to be treated).

The term "exponential" is used to indicate that the rate of change increases or decreases with time such that on a logarithmic plot, the relationship is linear. These pathologic, functional, and ESKD data are all compatible with native kidneys losing structure/function exponentially and having a half-life approximately $100-140$ years, as predicted by podometric analysis.

The slope of podocyte depletion versus age in native kidneys is similar to the slope of allograft survival versus donor age. Older donor age is powerfully associated with shorter allograft half-life (20). Linear reduction in podocyte density with age is a potential mechanism that could help explain this relationship. Death-censored deceased donor allograft half-life versus age was therefore determined from the Organ Procurement and Transplantation Network/United Network for Organ Sharing (OPTN/UNOS) database (25) as shown in Table 1 and Figure 4. The standardized slope shown for allograft half-life versus age in Figure 4 was not statistically significantly different from the slope for podocyte density decrease versus age in Figure $2(P=$ $0.4)$. This result is therefore consistent with podocyte density being related to allograft half-life in some way such that at time of kidney donation, the podocyte density plays either a direct or indirect role in determining allograft half-life.

Simulation of allograft half-life from podometric data. To further test the hypothesis that podometric 
Table 1. Death-censored graft half-life for deceased donor kidney allografts performed in the United States between October 1987 and June 2013 (data as of September 5, 2014)

\begin{tabular}{|c|c|c|c|}
\hline & Donor age (yr) & $n$ & Half-life $(y r) \pm 95 \% \mathrm{Cl}$ \\
\hline & $15-24.9$ & 26,555 & $18.0(17.6-18.9)$ \\
\hline & $25-34.9$ & 18,951 & $16.7(15.9-17.5)$ \\
\hline & $45-54.9$ & 29,960 & $12.7(12.4-13.1)$ \\
\hline & $55-64.9$ & 18,292 & $11.0(10.7-11.3)$ \\
\hline & $65-74.9$ & 4,626 & $9.0(8.5-9.3)$ \\
\hline
\end{tabular}

Data from the OPTN/UNOS database (25) restricted to first-time kidney transplants. $n=$ number of allografts used for the calculations. Half-life data are shown as the median $\pm 95 \%$ range (in parentheses). Cl, confidence interval.

changes might be related to allograft half-life, we combined podometric data from aging and allograft studies to attempt to predict the relationship between allograft survival and age. We previously reported that at time of transplantation, podocyte density decreased on average by $20 \%$ due to compensatory glomerular hypertrophy and that the detachment rate for allografts was on average 6-fold higher than in normal controls with two kidneys (19). The actual rate of podocyte detachment varied from being 2-fold increased above normal in long-lived stable allografts and 10- to 20-fold increased in transplant glomerulopathy. Therefore, as shown in Table 2, to simulate events in allografts we estimated the projected podocyte density that would be present at any designated age, projected a further $20 \%$ reduction in podocyte density to occur at the time of transplantation, and that this would be followed by an average 6-fold increased rate of podocyte detachment until podocyte density reached a value of 50 per $10^{6}$ $\mu \mathrm{m}^{3}$, which would represent ESKD (see Figure $5 \mathrm{~A}$ ). This value of 50 per $10^{6} \mu \mathrm{m}^{3}$ representing ESKD is derived from prior studies in allografts (19). The calculated slope for hypothetical allografts donated at various ages was not statistically different from the conditional allograft half-life values observed in OPTN/UNOS data as shown in Figure 5B. Furthermore, at the average age of a kidney donor (40.1 years), the simulation predicted an allograft half-life of 15.1 years, which is close to the observed value of 14.2 years (Table 1). These data are compatible with the hypothesis that changes in podocyte density and rate of loss reflect the events taking place in the average allograft.

The donor age effect and accelerated graft loss of function. To further test this hypothesis, we performed additional slope analysis using death-censored average allograft half-life values. The relationship between donor age and allograft half-life derived from Table 1 is replotted as shown in Figure 6A. The slope of the line is not 1.0, as would have been expected if the biologic aging rate in the donor and recipient were the same. Rather the slope is 5.6, indicating that allografts lose function 5.6-fold faster

Table 2. Simulation experiment to use podometric data to project time to end-stage kidney disease in relation to donor age

\begin{tabular}{|ccccccccc}
\hline Donor age $(\mathbf{y r})$ & $\mathbf{0}$ & $\mathbf{2 0}$ & $\mathbf{3 0}$ & $\mathbf{4 0}$ & $\mathbf{5 0}$ & $\mathbf{6 0}$ & $\mathbf{7 0}$ & $\mathbf{8 0}$ \\
\hline Density decrease at $-3.2 / \mathrm{yr}$ & 0 & 64 & 96 & 128 & 160 & 192 & 224 & 256 \\
\hline Density at donation time & 338 & 274 & 242 & 210 & 178 & 146 & 114 & 82 \\
\hline Density reserve above 50 & - & 224 & 192 & 160 & 128 & 96 & 64 & 32 \\
\hline Est. time to ESKD at -10.6/yr & - & 21.1 & 18.1 & 15.1 & 12.1 & 9.1 & 6.0 & 3.0
\end{tabular}

Row 1: Estimated average decrement in podocyte density calculated as -3.2 per $10^{6} \mu \mathrm{m}^{3}$ per year (see Table 1 in ref. 17). Row 2: Predicted podocyte density at each donor age obtained by subtracting the values shown in row 1 from 338 (the average projected podocyte density at birth; ref. 17). A podocyte density of 50 per $10^{6} \mu \mathrm{m}^{3}$ or below is associated with end-stage kidney disease [ESKD; ref. 19). Row 3 therefore shows the density reserve above 50 per $10^{6}$ $\mu \mathrm{m}^{3}$ at each donation age. From aging data, we know that each glomerulus loses on average 1.76 podocytes per year (17). From allograft data, we know that the rate of podocyte detachment after transplantation is 6-fold higher than the control median value (19). Therefore, the average rate of decrease in density after implantation is estimated to be $1.76 \times 6=10.6$ per $10^{6} \mu \mathrm{m}^{3}$ per year. Row 4 gives the estimated time to ESKD obtained by dividing the reserve density values in row 3 by 10.6 . 


\section{Glomerulosclerosis}
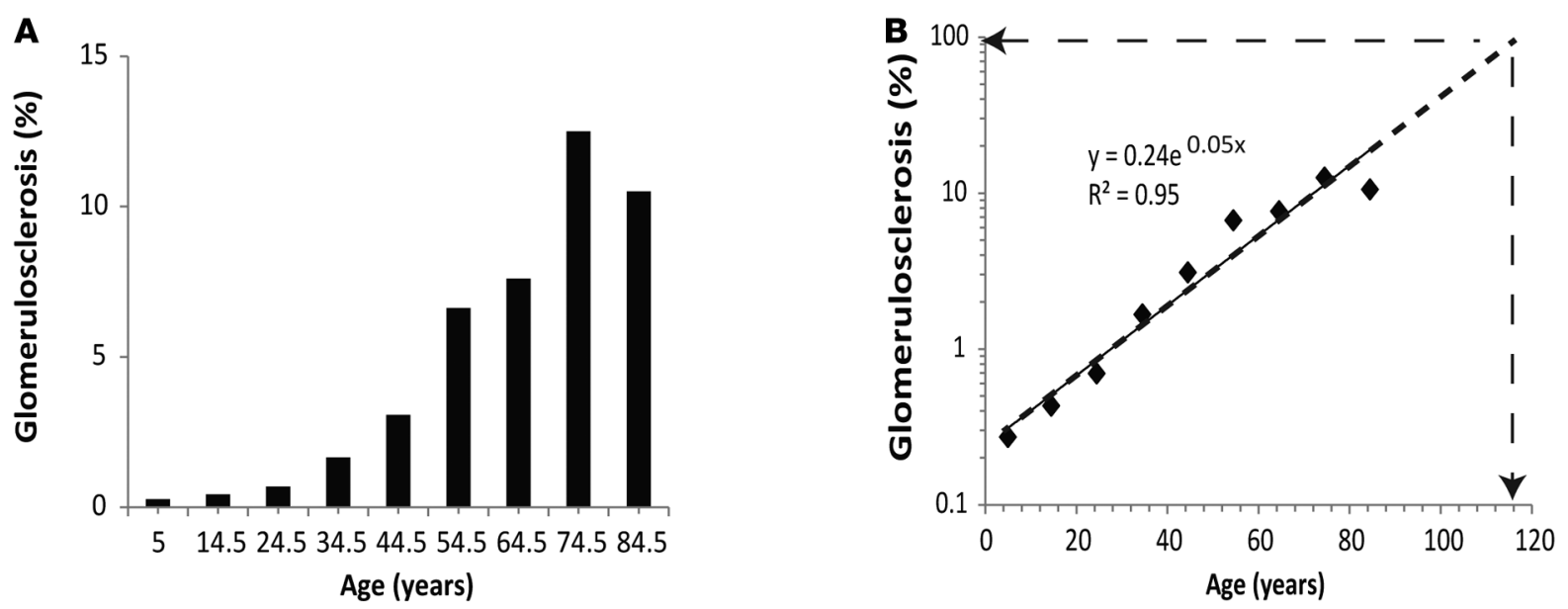

\section{Renal function}
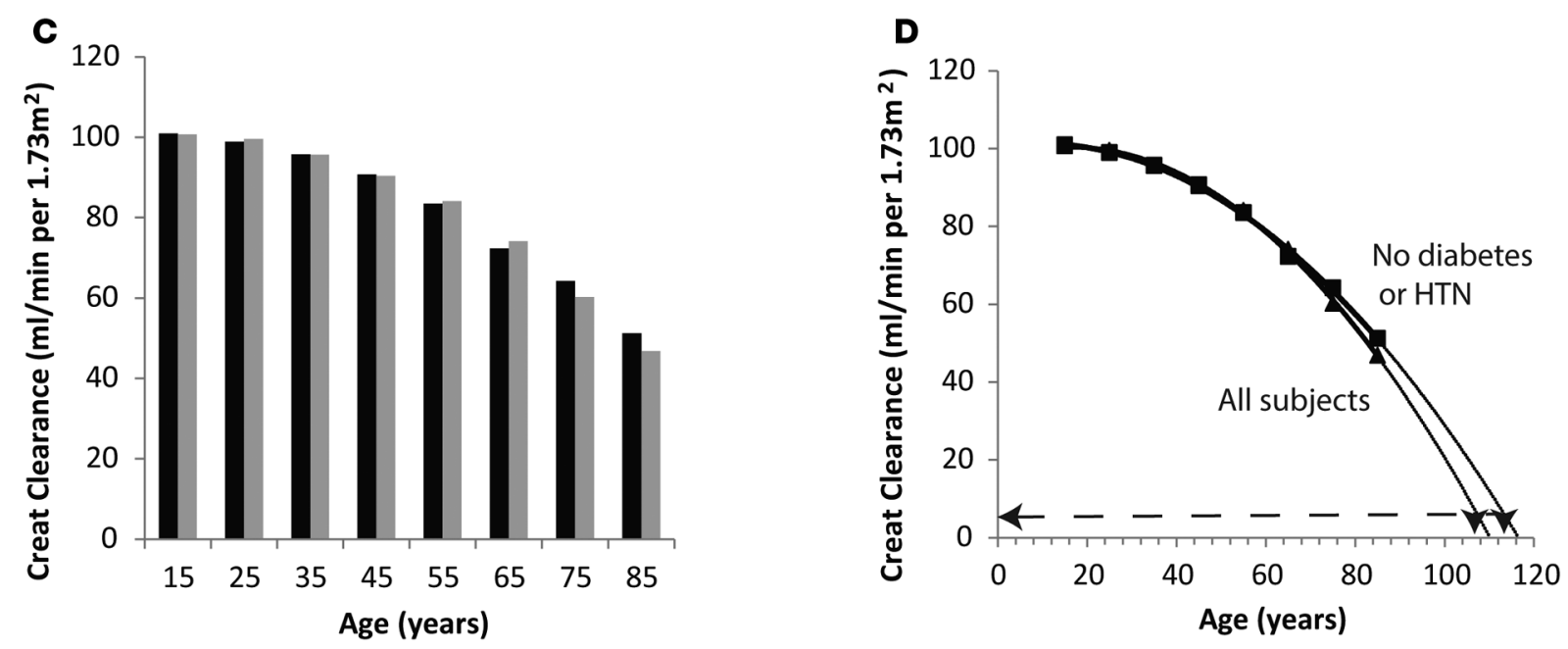

\section{Cumulative ESKD}
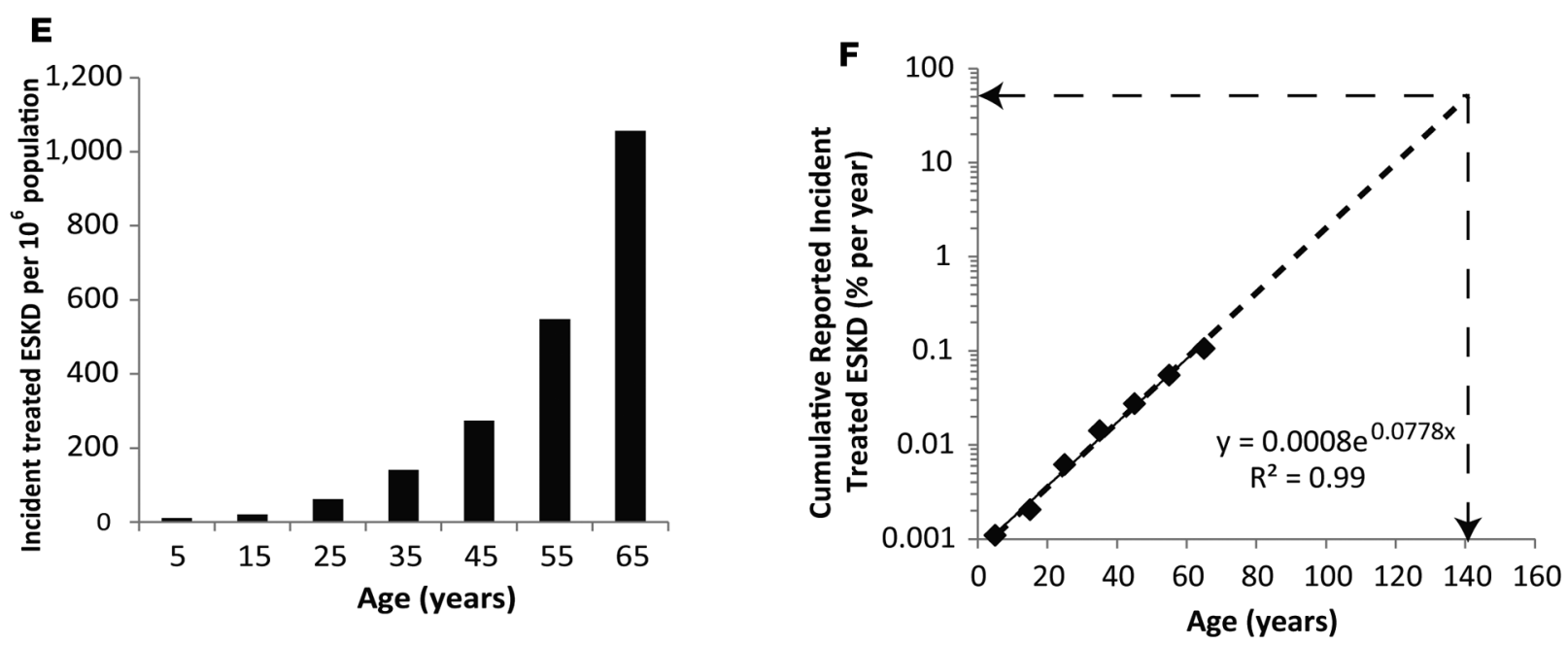
Figure 3. Native kidney half-life estimations. (A and B) Proportion of glomeruli with global glomerulosclerosis derived from Kaplan et al. (21). Meaned data for global glomerulosclerosis prevalence appears to increase exponentially with age. The dotted line is extended to determine the age at which $95 \%$ of glomeruli are projected to be sclerotic (presumed to be incident end-stage kidney disease [ESKD]), as shown by the dashed lines with arrows. This projected value is 116 years. (C and D) Estimated glomerular filtration rate (eGFR) projections from the NHANES dataset. eGFR was calculated as the creatinine (Creat) clearance per $1.73 \mathrm{~m}^{2}$. (C) Reduction in eGFR with age of all subjects (black bars) and only those subjects without diabetes or hypertension (gray bars). (D) The rate of decrease in eGFR accelerates with age such that the age at which eGRF is projected to reach $5 \mathrm{ml} / \mathrm{min}$ per $1.73 \mathrm{~m}{ }^{2}$ (presumed to be ESKD) for all subjects is about 107 years and for only those without diabetes or hypertension is about 110 years, as shown by the dotted lines with arrows. See supplemental material for details of fitting a quadratic polynomial equation and the derived mean projected age and ranges at ESKD. (E and F) Reported incident treated ESKD from the USRDS Annual Data Report (24). The number of incident aggregated treated ESKD per million of population is shown in $\mathbf{E}$, demonstrating an exponential increase with age. (F) Cumulative reported treated ESKD as percentage of the population plotted on a log scale in order to estimate the age at which $50 \%$ of the population would be projected to reach ESKD (i.e., the half-life of native kidneys), as shown by the arrows. The projected value is 140 years. The $r^{2}$ values shown are for aggregated data.

than donor kidneys. This 5.6-fold value is numerically comparable to the podometric analysis, which found an average 6-fold increased rate of podocyte detachment in allografts compared with native kidneys (19). Furthermore the $y$ intercept for this line is 121 years. Therefore, if the kidney was never transplanted (i.e., the allograft half-life was 0 years), the average life span of the donor kidney would be projected to be about 121 years, a value that approximates the average life span of native kidneys derived by four independent methods shown above.

The foregoing data lead us to posit an accelerated function loss concept for allografts, as shown in Figure 6B. An average kidney has a certain age at time of donation and then accumulates accelerated loss of function (equivalent to accelerated aging) as a transplant, so that its total expected average life span = kidney age at time of donation + allograft life span $\times 5.6=120$. According to this scenario, the projected cumulative average life span at which deceased donor allografts will fail is always 120 "biological" years (donor years + recipient years $\times 5.6$ ) regardless of donor age. The average projected life of a deceased donor kidney can therefore be estimated as (120 - donor age)/5.6. The large number of years of allograft function that is lost due to the accelerated aging-like process is illustrated by the number of years between the squares and circles at any donor age in Figure 6B.

\section{Discussion}

In this report we used available published data sources to test predictions derived from the observation that podocyte density decreases linearly with age. Remarkably, all outcome parameters tested are compatible with the predictions, including using two different and independent kidney cohorts (native kidneys and allografts). Although, as outlined in the Introduction, podocyte depletion is unequivocally proven to cause progression to ESKD, the relationships shown in this report are associations that do not themselves prove causation. Had these relationships been found for any one outcome parameter, they could have resulted from chance alone. However, the fact that the data are all internally consistent makes chance relationships less likely. The following discussion therefore assumes that they represent real biological processes and explores their implications.

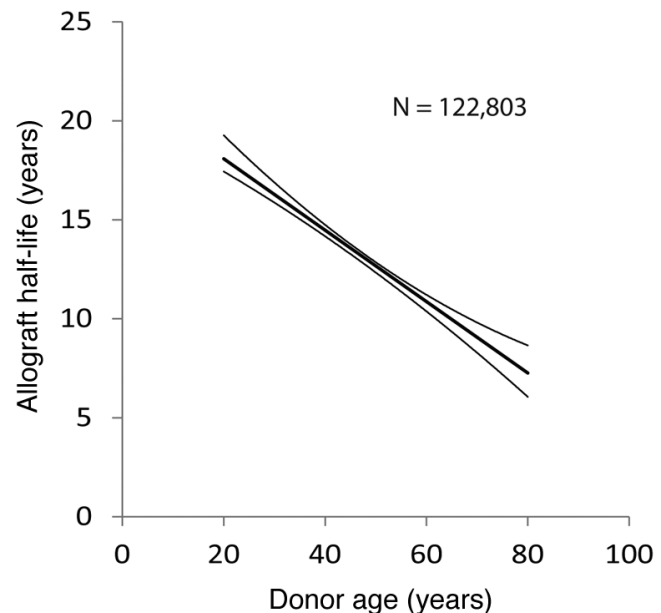

Figure 4. The slope of allograft half-life in relation to donor age. Data for death-censored donor allograft survival versus age were derived from the OPTN/UNOS database as of September 5, 2014, as outlined in Methods (25). The median and $95 \%$ confidence limits are shown together with the $n$. 
A

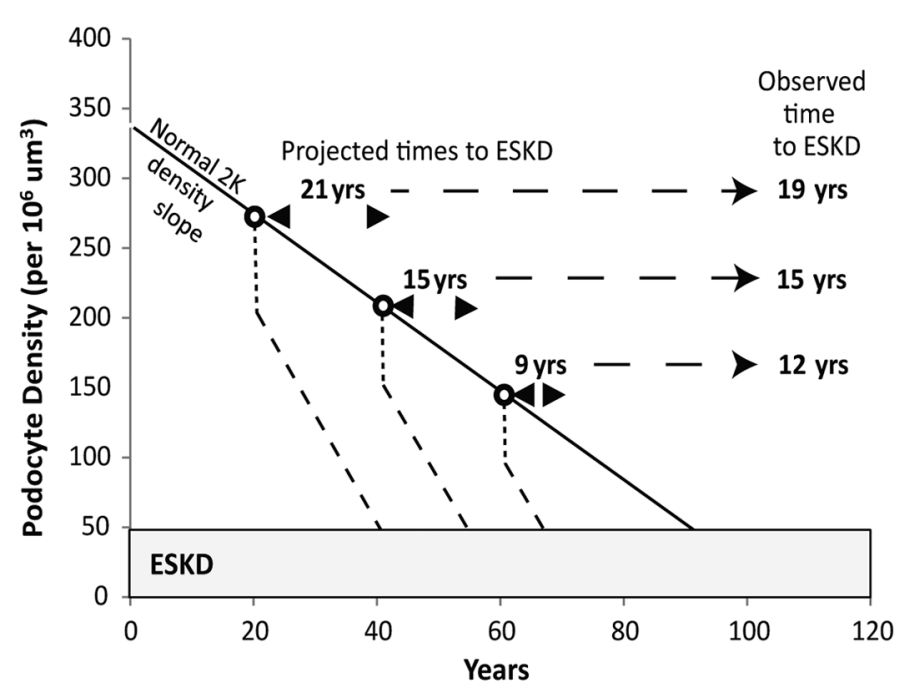

B

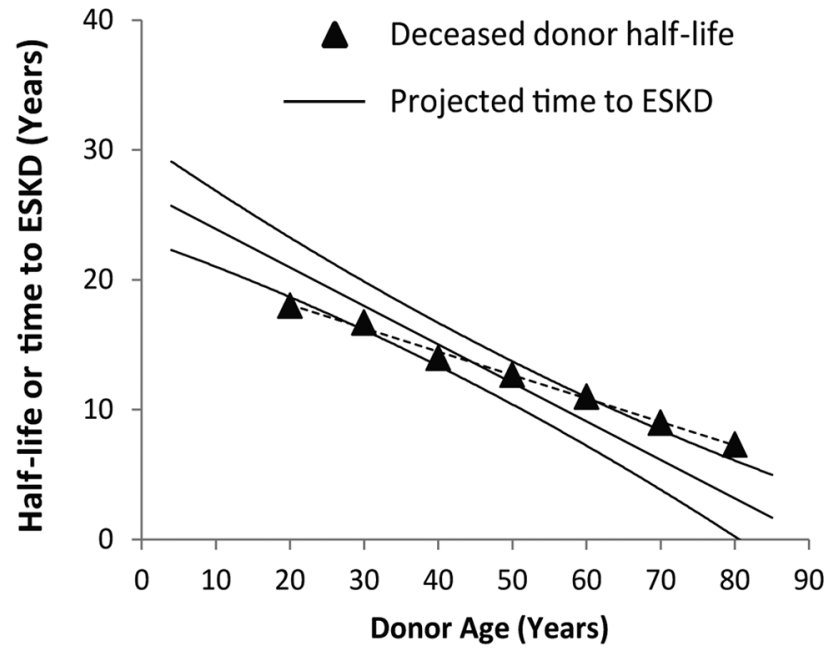

Figure 5. Simulation of allograft time to ESIKD versus donor age derived from podometric data and comparison to observed transplant outcomes data versus age. (A) Method and assumptions. The normal reduction in podocyte density by age is illustrated by the diagonal solid line as previously reported (17). Open circles represent hypothetical kidney donation ages at 20, 40, and 60 years. At implantation both the kidney and glomerulus rapidly increase in size by $20 \%$ (19). Podocytes cannot divide and therefore have to undergo rapid compensatory hypertrophy, resulting in reduced podocyte density, as shown by the dotted line at each donor age. Following implantation, allografts continue to lose podocytes at an increased rate on average 6 -fold above the native kidney control rate (19). This increased rate of podocyte detachment will result in an accelerated rate (steeper slope compared with native kidneys) of further reduction in podocyte density (dashed lines). At a podocyte density value below 50 per $10^{6} \mu m^{3}$, global sclerosis will supervene and kidneys will be at end-stage kidney disease (ESKD). The predicted time to ESKD can therefore be estimated for any putative donor age, as shown by the arrowheads. The observed mean value for time to ESKD for deceased donor kidney transplants at each of the three donor ages is shown at right as derived from the OPTN/UNOS database as of September 5, 2014. (B) Comparison of projected to observed kidney transplant halflife by age. Each data point $(n=89)$ from the previously reported aging study (17) was used to estimate the projected time to ESKD that would have occurred were that kidney to have been transplanted according to the above criteria. The regression line $\pm 95 \%$ confidence limit is shown. The closed triangles show aggregated half-lives by age for deceased donors as derived from the OPTN/UNOS database as of September 5 , 2014. The slope of the linear regression line derived from podometric data (solid line) is steeper than the observed OPTN/UNOS data, although this difference is not statistically significant $(P=0.42$; general linear model).

Although all cell types and kidney compartments participate in both aging and superimposed injury processes, podocytes can be considered as attractive candidates to reflect these complex biologic processes because (a) they are terminally differentiated, long-lived cells that are not readily replaced; (b) they express unique RNAs and proteins by which they can be recognized and quantitated; (c) they reside on the urinary space side of the glomerular basement membrane and therefore detach into the filtrate, where they can be measured in urine (unlike endothelial cells); (d) events causing endothelial injury (for example in preeclamptic toxemia [PET]) are signaled by podocyte detachment so that diverse forms of injury can potentially be read out by the podocyte detachment rate (26-29); (e) the circumscribed spherical structure of glomeruli makes it easy to define their borders and quantitate podometric parameters; and (f) the well-established relationship of podocyte depletion to glomerular failure means that changes in podocyte density and rate of loss per se determine glomerular survival, such that what is measured is mechanistically related to outcome (4).

Two testable predictions result from the previously reported observation that podocyte density decreases linearly with age, eventually resulting in critical podocyte depletion, mass podocyte detachment, glomerular tuft collapse, and global glomerulosclerosis (17). First, changes in structure (global glomerulosclerosis), function (eGFR per $1.73 \mathrm{~m}^{2}$ ), and ESKD are all found to be exponentially related to age. Second, the average life span for a human kidney projected from four different approaches (podometrics, \% glomerulosclerosis, eGFR, and incident ESKD) is estimated to be in the range of 100-140 years. Kidneys can thus be viewed as being designed to have an average life span of about 100-140 years, analogous to an automobile component that is engineered to last long enough to survive the average life span of the vehicle. In the kidney a "weak link" in the design that may limit life span is the podocyte and its inherent biology. Most people living an average life span (about 78 years in the United States) would 
A

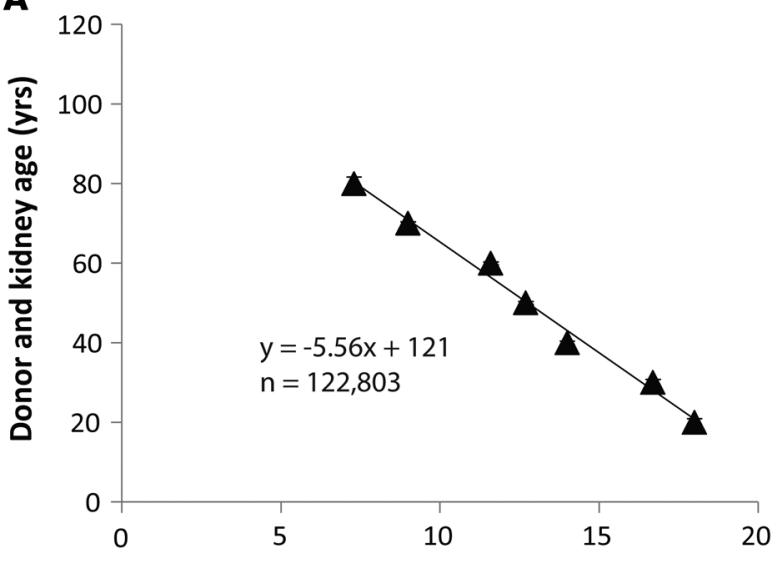

Observed allografthalf life (yrs)
B

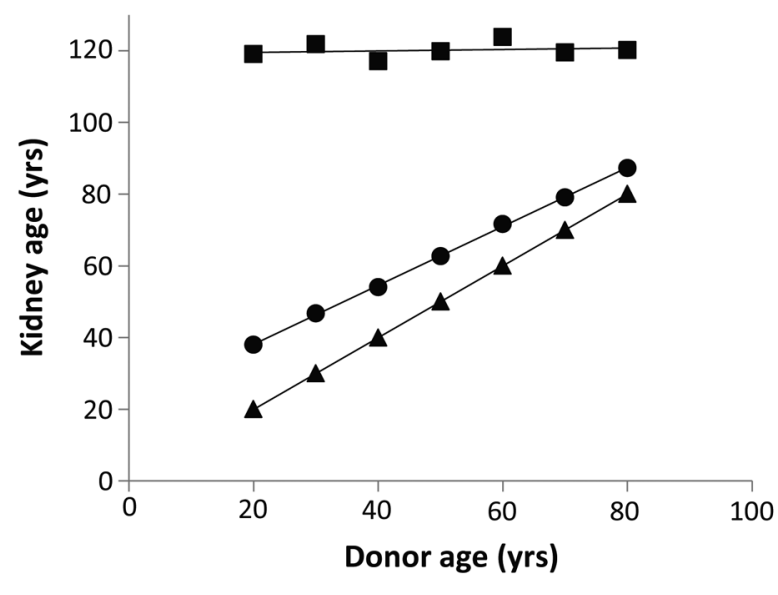

Figure 6. Donor age in relation to allograft half-life plots. (A) Donor age versus allograft half-life. Deceased donor allograft half-life is plotted on the $x$ axis and kidney age on the $y$ axis from data shown in Table 1. Donor kidney age is linearly related to allograft half-life over all donor ages from 20 to 80 years $(P$ $<0.001$ for aggregated data, $n=122,803$; linear regression model). The slope coordinates can be interpreted to show that the allograft aging rate is 5.6 -fold faster than the donor aging rate, and that had the kidney not been transplanted (half-life = 0 years), its expected life span would have been about 121 years. (B) "Biologic" versus "chronologic" kidney aging. Donor age is plotted on the $x$ axis and total kidney age (either chronologic or biologic) on the $y$ axis. The triangles represent the kidney age at time of donation. The circles show the average total chronologic kidney age at which the allograft will fail for various donor ages (estimated as the kidney age at donation + the chronologic allograft half-life). The squares show the total biologic kidney age at which the kidney will fail (estimated as the kidney age at donation + the allograft half-life $\times 5.6$ to account for accelerated allograft biologic aging). Thus, all allografts, whatever their donor age, will on average fail when their total biologic age (donor age + allograft age $\times 5.6$ ) $=121$ years. The difference between the squares and the circles at any donor age represents the lost allograft function due to the accelerated aging-like process experienced by allografts that could be targeted therapeutically. Data are from the OPTN/UNOS database as of September 5,2014 (25).

not experience ESKD unless they were subjected to superimposed accelerated podocyte depletion (due to a genetic susceptibility or injury process) such that their 100- to 140-year kidney life span capacity becomes exhausted prior to death from some other cause. On the other hand, as the average life span of a population increases, the prevalence of ESKD will be expected to exponentially increase, unless the biology of the system is altered in some way.

Podocyte depletion fulfills all three criteria defined as a "hallmarks of aging" by Lopez-Ortin and colleagues (30): (a) podocyte depletion is manifest during normal aging (17, 31); (b) experimental podocyte depletion accelerates aging-associated glomerulosclerosis (5, 6, 29, 31); and (c) experimental amelioration of podocyte depletion (accomplished by various methods but particularly by the classic age-extension paradigm of calorie restriction) retards podocyte depletion and hence increases (kidney) life span $(7,31)$. Podocytes themselves will also undergo epigenetic and functional changes with age that make them more susceptible to hypertrophic stress. According to this scenario, at a normal aging rate human kidneys would consume their reserves to reach ESKD after an average 100-140 years. The extent to which podocyte reserves have been consumed at any point in time ("biologic age") would be represented by the measured podocyte density. The rate at which the accelerated aging process is proceeding at any point in time ("biologic aging rate") would be represented by the podocyte detachment rate. Although many other aging processes represented by other hallmarks of aging will also occur in the kidney in parallel to podocyte depletion and will represent alternative methods of measuring kidney aging, for the reasons outlined above podocyte depletion represents a potentially practical approach for understanding aging-like processes in the kidney that impact kidney life span (4). This concept can be further tested by application to an independent kidney failure cohort, namely kidney allografts.

Taking into account the linearly decreasing podocyte density with age, we used podometric data from allografts to simulate within reasonable bounds the actual relationship between age and death-censored deceased donor graft outcome derived from OPTN/UNOS data. This quantitatively accounts for both the donor age effect and the average allograft survival at about 15 years, and could also help explain why long-term allograft survival has not improved substantially in recent eras despite remarkable success in the transplant community in improving short-term allograft outcomes $(32,33)$.

Net kidney allograft half-life is thought to be limited by a combination of injury processes, including 
ischemia/reperfusion injury, immune events, drug toxicities, infections, diabetes, hypertension, recurrent diseases, and other insults that collectively impact structure and function, resulting in a progressive fibrotic phenotype (34). Recent studies have emphasized the importance of immune-mediated microvascular injury (34-37). The finding that the rate of podocyte detachment appears to provide a measure of this complex interacting biology is, at first sight, surprising. However, if mechanisms of allograft injury that are truly determinative of long-term outcome (such as immune-mediated microvascular injury) are also reflected by podocyte detachment, then this result may not be incongruous. PET is a prototypical human endothelial injury process whereby angiogenic factors produced by the placenta impact endothelial cells, particularly of the glomerulus, to drive primary endothelial injury. There is now substantial literature indicating that podocyte detachment rate as measured in urine is a sensitive and specific marker for PET in the pregnancy setting, and degree of podocyte loss correlates with longer-term outcome (26-29). By analogy, processes driven by microvascular injury in the allograft could also drive podocyte detachment rate in proportion to endothelial injury. Similarly, other disease processes active in allografts, such as diabetes, hypertension, obesity, and recurrent glomerular disease, would be expected to operate through accelerated podocyte detachment (1-4). As we previously reported, the measured rate of podocyte detachment in individual allografts varies quite widely, with long-lived allografts having lower levels and transplant glomerulopathy associated with high levels (19). Further studies will be required to determine whether these differences can provide actionable clinical information for individual patients. However, it is important to note that some injury mechanisms that commonly contribute to allograft failure will likely not be reported out through podocyte detachment rate, particularly those impacting the tubule-interstitial compartment in early graft life. Markers for immune processes, drug toxicities, ischemia/reperfusion injury, recurrent disease, and vascular processes known to impact graft outcome will more reliably capture these processes for an individual patient.

While podocyte depletion could serve as a passive readout for microvascular injury and other immune and non-immune processes thought to drive allograft failure, an alternative hypothesis would be that hypertrophic podocyte-dependent processes themselves might contribute to shortening long-term allograft life span. If this hypothesis could be substantiated, it would offer alternative nonimmune strategies toward prolonging allograft survival. One question is therefore whether the characteristic clinical features of longterm allograft failure are compatible with a podocyte-dependent process contributing some element of allograft failure. Allograft outcome is well known to be associated with increased levels of proteinuria (38-40). Halloran and colleagues previously suggested that chronic allograft nephropathy resembled aging kidney and that an accelerated aging mechanism ("senescence") and nonimmune factors play a significant role in allograft failure (41). Chronic allograft changes are illustrated by the landmark 2004 study by Nankivell and colleagues, who performed protocol biopsies in allografts where the underlying disease (type 1 diabetes mellitus) that had caused native kidney failure had been cured by pancreas transplantation (42). They observed that tubule-interstitial injury associated with immune/inflammatory events was prevalent in the first years after transplantation. However, by 6-10 years the most obvious lesions were global glomerulosclerosis affecting 37\% glomeruli associated with tubule-interstitial and vascular hyalinosis lesions. El-Zoghby and colleagues also reported a major glomerular contribution to long-term allograft failure (43). Pathologic markers characteristic of transplant glomerulopathy such as reduplication of basement membranes are also features of PET and membranoproliferative glomerulonephritis, where podocyte depletion will likely play a role. As is well known from studies by both Risdon and Bohle and colleagues, the most robust pathologic evidence for progression in primary glomerular disease is interstitial fibrosis, and degree of interstitial fibrosis correlates with degree of kidney dysfunction, while degree of glomerulosclerosis correlates to a lesser extent $(44,45)$. Kaissling, LeHir, and Kriz show mechanistically how podocyte-induced glomerular failure leads to tubule-interstitial fibrosis $(2,46)$. Globally sclerosed glomeruli involute and become increasingly difficult to recognize, such that their contribution to the progression process becomes difficult to discern. Reduction in interstitial capillary density, interstitial fibrosis, and tubular atrophy (IF/TA) are also major pathologic processes associated with long-term allograft failure, reflecting the experience of the allograft in surviving ischemia/reperfusion injury, as well as immune-mediated and calcineurin inhibitor toxicity events. Graft injury by any mechanism is likely to result in heightened allogeneic immune responses that drive further immune-mediated microvascular injury, making cause and effect difficult to resolve. In summary, these data do not exclude an unrecognized hypertrophic and/or podocyte-dependent mechanism contributing to allograft failure at some level. 
Data projections were required for this study because it is not possible to measure actual data from an "average" population 100-140 years old. The fact that projections from five different datasets provided comparable values for native kidney half-life suggests that the estimates are quite robust, but assumptions and biases are inherent in this analysis. For example, as aging proceeds, more and more people will die from various causes and not be available for counting (e.g., by the USRDS or by enumerating glomerulosclerosis in autopsy samples), resulting in underrepresentation of the true number of people who have reduced eGFR and reach ESKD at older ages. In contrast, those who do survive into old age are a highly select group of "successful" agers who are therefore not representative of the general population. This means that data from older age groups will tend to be biased, and that projections based on younger groups will more accurately reflect a representative view of the general population. Assumptions were also made that ESKD would occur on average when $95 \%$ of glomeruli are globally scarred and at an eGFR of $5 \mathrm{ml} /$ min per $1.73 \mathrm{~m}^{2}$. However, even if these assumptions are not strictly accurate, that would not significantly change the estimates for average kidney life span. The completeness and accuracy of database-derived estimates of half-life are subject to many limitations, including the inherent bias that the data used to measure half-life are, by definition, not representative of current practice. Therefore, current allograft management may not be represented by the half-life values used for this analysis, although available data do not support major improvements in long-term allograft outcome $(32,33)$.

We expect that environment can have significant effects on podometric parameters. For this report, podocyte density data in relation to age were obtained from people resident in the state of Michigan, which has high obesity levels (47). This means that glomerular volume, which is related to body mass index, may have been increased to a greater extent than in a national or international population sample, particularly in older age. This would tend to make podocyte density relatively lower and account for a somewhat steeper slope of podocyte density versus age compared with the allograft survival versus donor age data for the United States as a whole. Podocyte density, and thereby possibly also multifactorial glomerular aging, is therefore likely to be a rather plastic variable that might be quite susceptible to modification by diet and therapeutic approaches to attempt to change outcomes at the individual and population level for both native kidneys and allografts.

These data support a concept whereby linear reduction in density of long-lived postmitotic cells with aging requires parallel compensatory cell enlargement, leading eventually to critical hypertrophic cell stress, mass cell detachment, glomerular tuft collapse, and global glomerulosclerosis (18). As aging proceeds, a greater and greater proportion of glomeruli at any point in time will reach criticality and fail, thereby causing a clinical phenotype represented by exponentially changing structure/function with age and a limited organ half-life. This paradigm is potentially relevant to other aging-associated diseases, such as those affecting brain (neurons; Alzheimer and Parkinson diseases), pancreatic islets ( $\beta$ cells; type 2 diabetes), and heart (conducting cells; atrial fibrillation).

In summary, we provide support for the following concepts: (a) human kidneys are designed to have an average biologic age capacity estimated to be about 100-140 years, and their rate of aging can become accelerated under various conditions, such that ESKD supervenes before the end of an average life span; (b) kidney transplantation provides an independent cohort proof-of-concept example where allografts can be considered to undergo an accelerated aging-like process that quantitatively accounts for their unexpectedly short half-life, the remarkable donor age effect, and the fact that long-term allograft attrition rate has not improved much in recent years; and (c) podometrics can be used to estimate kidney biologic age at a point in time (podocyte density) and the rate at which the aging-like process is proceeding (podocyte detachment rate). This paradigm offers novel tools to monitor progression and identify targets for intervention.

\section{Methods}

Age-related incidence of globally sclerosed glomeruli in human kidneys. Data were obtained from the report by Kaplan et al. (21) as likely to be most representative of the general population over a wide age range. This autopsy dataset used 122 samples, each about $1 \times 2 \mathrm{~cm}$ sections of kidney cortex containing about 200 glomeruli per sample capable of providing a reasonably representative estimate of sclerotic glomeruli. For the Kaplan series, a sclerotic glomerulus was defined as "total or near total acellular global obliteration of the tuft." These authors evaluated 122 autopsy kidneys from two sources, including "medical examiner cases" with no known history of kidney disease and who had experienced sudden unexpected death due to trauma, narcotic overdose, anesthesia, alcoholism, acute medical illness, and unknown causes; and 
"hospital cases," who died in hospital with no history of hypertension or renal disease and who had normal heart weights. The combined data for males and females ( 86 males and 36 females) was averaged for each age range and plotted against age. At any age variance is quite large, as emphasized by these authors (21). We assumed that a kidney would be at end stage when $95 \%$ of glomeruli were globally scarred.

Estimation of renal function from the NHANES dataset. All available samples from years 2009-2012 in the NHANES dataset, including those older than 80 , were used $(n=11,318)$ for the age group plot. Only samples age $12-79(n=6,985)$ were used for the fitted curve analysis, because in the NHANES dataset $(22,23)$ ages older than 80 years are coded as " 80 ." Statistical outliers $(n=407)$ were excluded. 1,024 subjects had both diabetes and hypertension, and 6,895 subjects had neither diabetes nor hypertension. To avoid using a method to estimate eGFR that includes age as a factor, we used creatinine clearance $(\mathrm{CrCl})$ adjusted for body surface area as follows: $\mathrm{A}, \mathrm{W}$, and $\mathrm{H}$ denote age (in years), weight (in $\mathrm{kg}$ ), and height (in $\mathrm{cm}$ ) of a subject, respectively. Scr is the serum creatinine (mg/dl), and Ucr and Urate are the urine creatinine concentration $(\mathrm{mg} / \mathrm{dl})$ and the urine flow rate $(\mathrm{ml} / \mathrm{min})$. To allow comparison of the $\mathrm{CrCl}$ between people of different body sizes, a corrected version of the creatinine clearance was defined as $\mathrm{CrCl}$ in $\mathrm{ml} / \mathrm{min}$ per $1.73 \mathrm{~m}^{2}=\mathrm{CrCl} \times 1: 73 / \mathrm{BSA}=\mathrm{Ucr} / \mathrm{Scr} \times$ Urate $\times 1.73 /$ $\left(0.007184 \times \mathrm{W}^{0.425} \times \mathrm{H}^{0.725}\right)$. Analysis was performed for all subjects to represent the total population and also by excluding those with diabetes and/or hypertension (see supplemental material). We assumed that a person would have reached ESKD when the eGFR was equal to $5 \mathrm{ml} / \mathrm{min}$ per $1.73 \mathrm{~m}^{2}$.

Estimation of cumulative native kidney incident treated ESKD as a measure of kidney half-life. Values for incident treated ESKD were as reported to the USRDS (24). The age range from 10 to 65 years was used recognizing that at less than 10 years of age developmental processes predominate and that at more than 70 years of age a greater proportion of cases are likely to be missing from the USRDS data set because they die from cardiovascular diseases and/or in an ICU setting, or elect not to be treated and are therefore not reported to the USRDS. The value of $n=57,514$ for the most recent 2012 data set was reported in the 2014 USRDS annual report. Cumulative values were calculated by sequentially adding values for younger ages to each age group. The age at which $50 \%$ of the population would be projected to have ESKD was used as the native kidney projected half-life estimate.

Estimation of allograft half-life. OPTN/UNOS Standard Transplant and Research (STAR) files based on OPTN data as of September 5, 2014, with data reported through June 30, 2014, were used for the analysis (25). Deceased donor kidneys were used for analysis because living donor kidneys are likely to represent a biased cohort for study. A Kaplan-Meier analysis was used to assess impact of donor age on allograft outcomes. Donors were categorized into 10 -year increments: 15 to $<25,25$ to $<35,35$ to $<45$, 45 to $<55,55$ to $<65,65$ to $<75$, and $>75$ years of age. Recipients were first-time kidney-only transplant recipients aged 18-70 years, transplanted from October 1, 1987, to June 30, 2013. Data were censored for death and loss to follow-up. Standard criteria donors (SCDs) and extended criteria donors (ECDs) were combined to form a single deceased donor (DD) group. Graft half-life and conditional half-life estimates were computed using a "period" method. The period method constructs a Kaplan-Meier survival curve if the half-life of a cohort in a given year is observed. Otherwise, we constructed the survival curve using the data from the cohort for the observed part and used survival data from earlier cohorts for the rest. For example, the 2007 half-life estimate for kidney graft survival is based on observed and survival data from earlier cohorts. For patients who underwent transplant in 2007, we observed 6-year survival data through 2013. We extrapolated this survival curve to its half-life by using the observed seventh-year failure rate of the 2006 cohort as the seventh-year failure rate of the 2007 cohort, the eight-year failure rate of 2005 cohort as the eighth-year failure rate of the 2007 cohort, and so on. Conditional half-life estimates were similarly computed, but limited to patients whose grafts survived the first year.

Podometric data. These data are as previously reported $(17,19)$. In normal humans, podocyte density decreases with age given by the equation $y=-3.17 \times$ age +338 , projecting that the age at which $y=0$ will be 107 years (17).

Estimation of "projected time to ESKD" for comparison with observed deceased donor half-life values. Individual values for podocyte density in relation to age as reported previously $(n=89)$ were used (17). Each observed value was treated as though it represented a transplanted kidney by applying the data previously reported from analysis of allografts (19), as shown in Table 2 and Figure 4A. Specifically, each value was reduced by $20 \%$ to simulate the decrease in podocyte density occurring due to glomerular hypertrophy at implantation, and then the rate of podocyte detachment was assumed to occur at 6-fold higher than normal rate there- 
after until the podocyte density was decreased to a value of 50 per $10^{6} \mu \mathrm{m}^{3}(19)$. Time from hypothetical transplantation to ESKD was then used to calculate the linear regression and the $95 \%$ confidence limits as shown in Figure 4B.

Statistics. Best fit curve estimations were applied to illustrate the cumulative change in ESKD by age. Linear regression model as the best-fitted model illustrated the relationship between donor kidney age, chronological graft age, and biological graft age versus allograft half-life. The interaction of the linear slope of the observed time-to-ESKD using DD data versus donor age and the slope of projected time-to-ESKD using podometrics versus donor age was tested to explore the statistical difference in the slopes. The test shown in Figure 5 was performed by application of a general linear model, with half-life as the dependent variable and donor age as the predictor, and the two groups defined as donor age versus projected time to ESKD as fixed factors. Then the $P$ value for interaction term of donor age by fixed factor was calculated. For Figure 6A, the $P$ value was determined by testing the slope of a linear regression line of "donor kidney age" versus "observed allograft half-life." A $P$ value of less than 0.05 was considered to be significant.

Study approval. All data are from previously published reports for which Institutional Review Board approval is listed or from large, publicly available datasets from NHANES, USRDS, or OPTN/UNOS. The report by Kaplan and colleagues (21) is an autopsy report series published in 1975 from the New York University School of Medicine. Many of these cases were Chief Medical Examiner cases of the City of New York referred for sudden unexplained death. No information is provided on whether informed consent was obtained.

\section{Author contributions}

RCW coordinated all aspects of the studies described. FA, JBH, LW, and MK worked with RCW to develop and test podometric concepts for the studies described and the prior studies upon which the current report is based. ASN, DC, MS, and AO constituted the kidney transplant team that met with RW over a 12-month period to work out how podometric parameters could be matched to transplant outcomes data. ASN performed the transplant outcomes analysis using the OPTN/UNOS database for transplant patients. MS also participated in the published studies used to develop podometric concepts for kidney transplants. YL, MZ, and FW met with RCW and the Nephrology Kidney Aging group including MB and JEW over an 18-month period focusing on understanding native kidney outcomes data in relation to podometrics and aging. YL, MZ, and FW also performed NHANES data analysis. The manuscript was written primarily by RCW, ASN, FA, and MB, but with substantial input from all other authors.

\section{Acknowledgments}

R.C. Wiggins acknowledges the support of the NIH (grants R01 DK 102643 and R01 DK 46073) and University of Michigan George M. O’Brien Kidney Translational Core Center P30 DK081943. F. Afshinnia was supported by NIH grant 1K08DK106523-01. Support for this work through the Robert C. Kelsch Collegiate Professorship Fund is gratefully acknowledged. This work was also supported in part by the Health Resources and Services Administration (contract 234-2005-370011C). The content is the responsibility of the authors alone and does not necessarily reflect the views or policies of the Department of Health and Human Services, nor does mention of trade names, commercial products, or organizations imply endorsement by the US government.

Address correspondence to: Roger C. Wiggins, Department of Internal Medicine, University of Michigan, Ann Arbor, Michigan 48109, USA. Phone: 734.936.4813; E-mail: rwiggins@umich.edu.

1. Kriz W, Gretz N, Lemley KV. Progression of glomerular diseases: Is the podocyte the culprit? Kidney Int. 1998;54(3):687-697.

2. Kriz W, LeHir M. Pathways to nephron loss starting from glomerular diseases: insights from animal models. Kidney Int. 2005;67:404-419.

3. Wiggins RC. The spectrum of podocytopathies: a unifying view of glomerular diseases. Kidney Int. 2007;71(12):1205-1214.

4. Kikuchi M, Wickman L, Wiggins RC. Podometrics as a potential clinical tool for glomerular disease management. Semin Nephrol. 2015;35(3):245-255.

5. Wharram BL, et al. Podocyte depletion causes glomerulosclerosis: Diphtheria toxin-induced podocyte depletion in rats expressing human diphtheria toxin receptor transgene. J Am Soc Nephrol. 2005;16(10):2941-2952.

6. Fukuda A, et al. Angiotensin II-dependent persistent podocyte loss from destabilized glomeruli causes progression of end stage 
kidney disease. Kidney Int. 2012;81(1):40-55.

7. Fukuda A, et al. Growth-dependent podocyte failure causes glomerulosclerosis. J Am Soc Nephrol. 2012;23(8):1351-1363.

8. Brown EJ, Pollak MR, Barua M. Genetic testing for nephrotic syndrome and FSGS in the era of next-generation sequencing. Kidney Int. 2014;85(5):1030-1038.

9. Sadowski CE, et al. A single-gene cause in $29.5 \%$ of cases of steroid-resistant nephrotic syndrome. J Am Soc Nephrol. 2015;26(6):1279-1289.

10. Pagtalunan ME, et al. Podocyte loss and progressive glomerular injury in type II diabetes. J Clin Invest. 1997;99(2):342-348.

11. Meyer TW, Bennett PH, Nelson RG. Podocyte number predicts long-term urinary albumin excretion in Pima Indians with type II diabetes and microalbuminuria. Diabetologia. 1999;42(11):1341-1344.

12. Steffes MW, Schmidt D, McCrery R, Basgen JM. International Diabetic Nephropathy Study Group: glomerular cell number in normal subjects and type I diabetes patients. Kidney Int. 2001;59(6):2104-2113.

13. White KE, et al. European Study for the Prevention of Renal Disease in Type I Diabetes (ESPRIT): podocyte number in normotensive type I diabetic patients with albuminuria. Diabetes. 2002;51(ESPRIT):3083-3089.

14. Lemley KV, et al. Podocytopenia and disease severity in IgA nephropathy. Kidney Int. 2002;61(4):1475-1485.

15. Della Vestra M, Masiero A, Roiter AM, Saller A, Crepaldi G, Fioretto P. Is podocyte injury relevant in diabetic nephropathy? Studies in patients with type 2 diabetes. Diabetes. 2003;52(4):1031-1035.

16. Wang G, et al. Podocyte loss in human hypertensive nephrosclerosis. Am J Hypertens. 2009;22(3):300-306.

17. Hodgin J, et al. Kidney aging and focal global glomerulosclerosis. a podometric perspective. J Am Soc Nephrol. 2015;26(12):3162-3178.

18. Wickman L, et al. Urine podocyte mRNAs, proteinuria, and progression in human glomerular diseases. J Am Soc Nephrol. 2013;24(12):2081-2095.

19. Yang Y, et al. The two kidney to one kidney transition and transplant glomerulopathy: A podocyte perspective. J Am Soc Nephrol. 2015;26(6):1450-1465.

20. Meier-Kriesche HU, et al. Interaction between donor and recipient age in determining the risk of chronic renal allograft failure. J Am Geriatr Soc. 2002;50(1):14-17.

21. Kaplan C, Pasternack B, Shah H, Gallo G. Age-related incidence of sclerotic glomeruli in human kidneys. Am J Pathol. 1975;80(2):227-234.

22. Centers for Disease Control and Prevention. National Health and Nutrition Examination Survey Data: NANES 2009-2010. http://wwwn.cdc.gov/Nchs/Nhanes/Search/nhanes09_10.aspx, Accessed May 7, 2016.

23. Centers for Disease Control and Prevention. National Health and Nutrition Examination Survey Data: NANES 2011-2012. http://wwwn.cdc.gov/Nchs/Nhanes/Search/nhanes11_12.aspx, Accessed May 7, 2016.

24. United States Renal Data System. 2012 Annual Data Report. USRDS Web site. http://www.usrds.org/atlas12.aspx. Accessed April 25, 2016.

25. [No authors listed]. Special issue: OPTN/SRTR Annual Data Report 2014. Am J Transplant. 2016;16(S2):4-215

26. Weissgerber TL, Craici IM, Wagner SJ, Grande JP, Garovic VD. Advances in the pathophysiology of preeclampsia and related podocyte injury. Kidney Int. 2014;86(2):275-285.

27. White WM, et al. Persistent urinary podocyte loss following preeclampsia may reflect subclinical renal injury. PLoS One. 2014;9(3):e92693.

28. Kandasamy Y, Watson D, Rudd D. Biomarker of early glomerular injury in pre-eclampsia. Hypertens Pregnancy. 2015;34(4):391399.

29. Henao DE, Saleem MA. Proteinuria in preeclampsia from a podocyte injury perspective. Curr Hypertens Rep. 2013;15(6):600605.

30. Lopez-Otin C, Blasco M, Partridge A, Serrano M, Kroermer G. The hallmarks of aging. Cell. 2013;153(6):1194-1217.

31. Wiggins J, et al. Podocyte hypertrophy, "adaptation" and "decompensation" associated with glomerular enlargement and glomerulosclerosis in the aging rat: prevention by calorie restriction. J Am Soc Nephrol. 2005;16(10):2953-2966.

32. Lamb K, Lodhi S, Meier-Kriesche HU. Long term renal allograft survival in the United States: a critical reappraisal. Am J Transplant. 2011;11(3):450-462.

33. Dharnidharka VR, Lamb KE, Zheng J, Schechtman KB, Meier-Kriesche HU. Lack of significant improvements in long-term allograft survival in pediatric solid organ transplantation: a US national registry analysis. Pediatr Transplant. 2015;19(5):477-483.

34. Venner J, Famulski K, Reeve J, Chang J, Halloran P. Relationships among injury, fibrosis, and time in human kidney transplants. JCI Insight. 2016;1(1):e85323.

35. Einecke G, et al. Antibody-mediated microcirculation injury is the major cause of late kidney transplant failure. Am J Transplant. 2009;9(11):2520-2531.

36. Sellarés J, et al. Understanding the causes of kidney transplant failure: the dominant role of antibody-mediated rejection and nonadherence. Am J Transplant. 2012;12(2):388-399.

37. Loupy A, et al. Molecular microscope strategy to improve risk stratification in early antibody-mediated kidney allograft rejection. J Am Soc Nephrol. 2014;25(10):2267-2277.

38. Amer H, Cosio FG. Significance and management of proteinuria in kidney transplant recipients. J Am Soc Nephrol. 2009;20(12):2490-2492.

39. Serón D, Burgos D, Alonso A. Histology and proteinuria after renal transplantation. Transplant Rev (Orlando). 2012;26(1):20-26.

40. Soler MJ, Riera M, Gutierrez A, Pascual J. New options and perspectives for proteinuria management after kidney transplantation. Transplant Rev (Orlando). 2012;26(1):44-52.

41. Halloran PF, Melk A, Barth C. Rethinking chronic allograft nephropathy: the concept of accelerated senescence. J Am Soc Nephrol. 1999;10(1):167-181.

42. Nankivell BJ, Borrows RJ, Fung CL, O'Connell PJ, Allen RD, Chapman JR. The natural history of chronic allograft nephropathy. N Engl J Med. 2003;349(24):2326-2333.

43. El-Zoghby ZM, et al. Identifying specific causes of kidney allograft loss. Am J Transplant. 2009;9(3):527-535.

44. Risdon R, Sloper J, De Wardener H. Relationship between renal function and histological changes found in renal biopsy speci- 
mens from patients with persistent glomerular nephritis. Lancet. 1968;17(7564):363-365.

45. Bohle A, Mackensen-Haen S, von Gise H. Significance of tubulointerstitial changes in the renal cortex for the excretory function and concentration ability of the kidney: a morphometric contribution. Am J Nephrol. 1987;7(6):421-433.

46. Kaissling B, LeHir M, Kriz W. Renal epithelial injury and fibrosis. Biochim Biophys Acta. 2013;1832(7):931-939.

47. Obesity Prevalence Maps. Centers for Disease Control and Prevention Web Site. http://www.cdc.gov/obesity/data/prevalencemaps.html. Updated September 11, 2015. Accessed May 5, 2016. 